\title{
Maximizing lon Transmission from Atmospheric Pressure into the Vacuum of Mass Spectrometers with a Novel Electrospray Interface
}

\author{
Andrew N. Krutchinsky, Júlio C. Padovan, Herbert Cohen, Brian T. Chait
}

Laboratory for Mass Spectrometry and Gaseous Ion Chemistry, The Rockefeller University, New York, NY 10065, USA

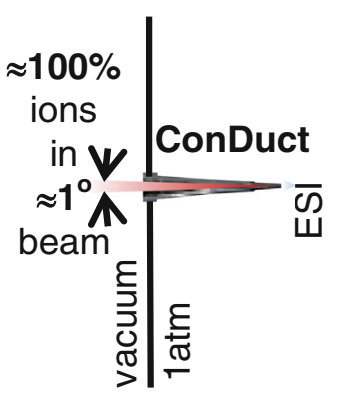

new interface transmits at least 400 times more ions than the commercial Thermo LCQ DECA XP atmosphere-tovacuum interface and 2 to 3 times more than the commercial interface in the Thermo Velos Orbitrap and the $Q$ Exactive mass spectrometers. We conclude that it might be possible to optimize the properties of the transmitted ions further by manufacturing ConDuct inlet electrodes from metal rather than conductive plastic and by determining the optimum angle of channel divergence and channel length.

Keywords: Electrospray ionization, ESI, Novel electrospray interface, Ion transmission, Atmosphere to vacuum ion interface, Mass spectrometry, Peptide ions, Conical duct, ConDuct

Received: 7 July 2014/Revised: 2 December 2014/Accepted: 3 December 2014/Published Online: 15 January 2015

\section{Introduction}

$\mathrm{I}$ deally, if every molecule in a sample could be converted into an ion and analyzed by mass spectrometry (MS) without loss, we would approach sample analysis with unit efficiency. In reality, the best reported efficiencies are in the range 0.01 $-0.1 \%$ [1-3], and outside of these special cases, the efficiencies are often much lower $(0.001-0.00001 \%)$ [4-6]. Thus, despite remarkable advances in $\mathrm{MS}$, there is still much room for improvement.

The majority of the ion losses usually occur during the first few steps of MS analysis, which include sample ionization in an electrospray (ESI) ion source, transfer of the ions into the entrance vacuum chamber of a mass spectrometer, elimination of cluster ion formation during the ion beam expansion process,

Electronic supplementary material The online version of this article (doi:10.1007/s13361-014-1062-1) contains supplementary material, which is available to authorized users.

Correspondence to: Brian T. Chait; e-mail: chait@rockefeller.edu and focusing and transfer of the ion beam into the mass analyzer. Recent technological advances have greatly reduced ion losses associated with some but not all of these steps.

The introduction of nano-ESI ionization dramatically increased the sensitivity of mass spectrometers [7-12] through improvements in the efficiency of sample ionization, scaled down requirements in sample solution flow-rate, and the lower potential needed for production of ions. These changes allowed researchers to move the tip of the ESI spray emitter closer to the entrance of the mass spectrometer, where the velocity of gas sucked into the inlet is the greatest. However, the resulting improved efficiency of ion entrainment does not automatically guarantee an increase in the efficiency of ion analysis because a large portion of the ion current may still consist of small droplets and ion clusters that are formed during the ESI process and collisional cooling of the gas and entrained material as they expand into the vacuum.

The efficiency of ion transmission and desolvation varies greatly in different types of atmosphere-to-vacuum interfaces. For example, ion beams produced by gas expansion through orifice-based interfaces tend to be quite divergent and dispersed 
[13]. To minimize transmission losses, the resulting beams are either collimated by positioning a skimmer close to the orifice $[14,15]$ or directed to an ion guide [16]. However, such configurations provide poor provision for supplying heat to the small residual charged droplets and solvated ions that are usually present. Several types of ion manipulation or modification to orifice-based interfaces can improve the ion desolvation process. These include increasing the declustering potential [17], introducing a counterflow of heated gas (sometimes referred to as a heated gas curtain) [18-20], heating the entire interface $[19,20]$, or installing a heated laminar flow chamber (particle discriminator interface) in front of the inlet orifice [2].

One efficient solution to the ion desolvation problem was advanced by our group in 1990 [21], where ions were introduced into the entrance vacuum chamber of the mass spectrometer via a heated metal capillary. Its length (approximately $20 \mathrm{~cm})$ and inner diameter $(\leq 0.5 \mathrm{~mm})$ were chosen to obtain subcritical Reynolds numbers for the gas flow in the capillary [22] to ensure a laminar flow, free of the turbulence that results in ion loss. Our group demonstrated that heat from the walls of the capillary caused efficient desolvation of ions prior to analysis in the mass spectrometer. Such ion interfaces containing a heated metal capillary, glass capillaries [23, 24]—or even an array of capillaries $[6,25]$ instead of a simple orifice - became widely adopted by MS manufacturers and researchers, especially when ESI ion sources with high flow rates were coupled to mass spectrometers.

Ion beams that expand into the vacuum through a capillary are also quite divergent and dispersed (see below). To collect ions from such divergent beams, Smith and coworkers replaced the skimmer with an ion guide consisting of multiple ring electrodes of decreasing diameters, which they termed an "ion funnel" [6, 26, 27]. Using this ion funnel, they were able to detect from $3 \%$ to $4 \%$ of the ions originally produced from the ESI solution [6]. Several commercial mass spectrometer manufacturers quickly followed this trend, either incorporating the original ion funnel or devices that serve a similar purpose.

Despite the unique qualities of metal capillaries as inlet electrodes, their suboptimal ion transmission efficiency remains one of the weakest links in the sequence of recent innovative improvements. Metal capillaries with wider diameters (up to $0.6 \mathrm{~mm}$ ) and shorter length (approximately $6 \mathrm{~cm}$ ) can transmit as much as $25 \%$ of the total ion current emanating from an ESI emitter tip depending on parameters such as the ESI potential and distance between the ESI emitter tip and the capillary $[5,28]$. To obtain additional improvements in the ion transmission efficiency, the length of the metal could be further shortened, but this likely comes at the expense of efficient ion desolvation [28]. These observations raise the question as to whether there are other ways to modify a capillary so as to increase its transmission efficiency and at the same time preserve the convenience of applying desolvation heat through the walls of the capillary.

Here, we report such a configuration. We discovered that an electrode containing a slowly diverging conical duct, which we termed the "ConDuct" electrode for convenience, can transmit approximately $100 \%$ of the total emitted ESI ion current into the vacuum. In addition, the ConDuct produces a narrow, very slowly expanding ion beam, with an angle of divergence of approximately $0.012 \mathrm{rad}$ (i.e., less than $1^{\circ}$ ), propagating in vacuum over long distances with very little increase in beam diameter. Intrigued by these properties, we constructed a novel ion transmission interface based on the ConDuct electrode and devised a technique for measuring its transmission efficiency. To implement the technique, we modified a commercial ion trap instrument (Thermo LCQ DECA XP) and equipped it with two atmosphere-to-vacuum interfaces - one that incorporates the new ConDuct inlet electrode while the other uses different reference interfaces that are present in commercially available mass spectrometers. We evaluated the relative transmission efficiency of the ConDuct interface versus the reference interfaces by comparing the signal intensities of spectra obtained from isotopically labeled versus unlabeled peptides entering the mass spectrometer through the two different interfaces.

\section{Experimental}

Figure 1a illustrates the experimental setup that we assembled to measure the efficiency of ion transmission from a nanospray ion source into the vacuum through a variety of different electrodes. It consists of an in-house constructed vacuum chamber, an ESI source facing an inlet electrode through which ions enter the vacuum, and an electrometer that can measure the transmitted ion current collected in a Faraday cup as well as the current lost during the ion transmission process. The majority of the results reported here are for three types of inlet electrodes: a ConDuct electrode made out of a $0.1-10 \mu \mathrm{L}$ capacity conductive plastic pipette tip (Advion, Ithaca, NY, USA, Figure 1b), a metal capillary from a commercial Velos ion trap mass spectrometer (Thermo Fisher Scientific, San Jose, CA, USA) (Figure 1c), and a 0.06-mm thick flat electrode containing a $0.6-\mathrm{mm}$ diameter orifice (Figure 1d). The description of the ESI sources, the equipment used to measure the ESI ion current, and other details of the experiments are given in the Supplementary Material.

Figure 1e shows a schematic of the modified LCQ DECA XP mass spectrometer, which we equipped to operate with two atmosphere-to-vacuum interfaces that accept ions from two different ion sources. This configuration allowed us to compare the relative transmission efficiencies of one interface relative to another. To do this, we first removed the original interface of the mass spectrometer and replaced it with a custom-made quadrupole ion guide $q_{1}$, an ion focusing electrode $e_{1}$, and a radio frequency (RF)-only T-shaped quadrupole ion guide $q_{T}$ operating in rf-only mode. The T-quadrupole enables the mass spectrometer to operate with two ion sources simultaneously $[29,30]$. To the right-hand side of the T-quadrupole arm, we attached a custom-made interface utilizing a ConDuct inlet electrode mounted at one end of a $30-\mathrm{cm}$ long pipe with inner diameter of $1.0 \mathrm{~cm}$ through a ball valve (to allow rapid 

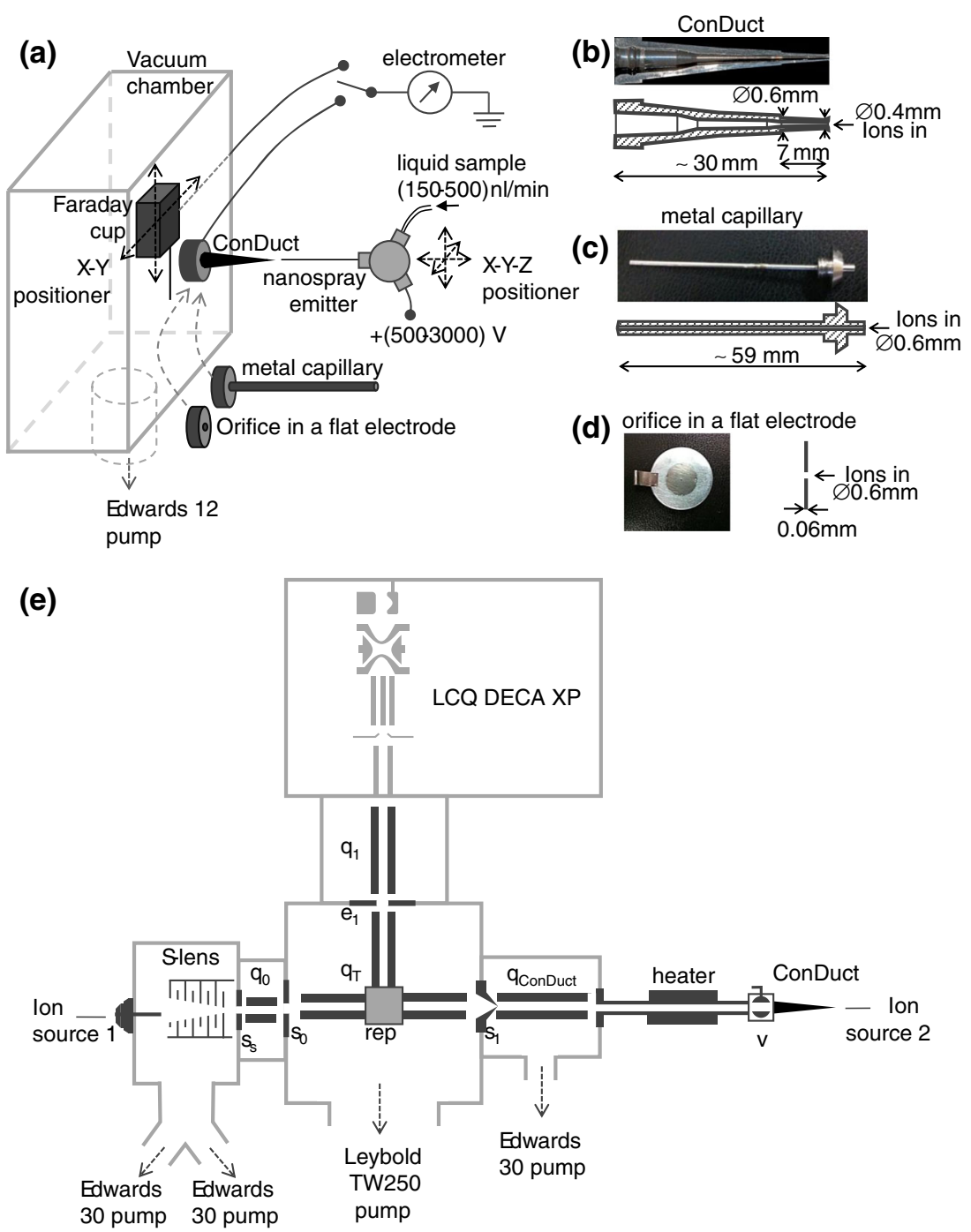

Figure 1. Experimental setups used in the present work. (a) Setup used to measure the transmission efficiency of ion current through the different electrodes. The configuration shown is for the measurement of the ion transmission efficiency of (b) a ConDuct electrode, but it can be quickly replaced with (c) a metal capillary electrode or (d) a flat electrode with an orifice. (e) Schematic of a modified LCQ DECA XP mass spectrometer with a recent Thermo S-lens interface (left-hand side) and the ConDuct interface (righthand side)

replacement of the ConDuct tip). The temperature of the center of the pipe was raised to $100-200^{\circ} \mathrm{C}$ by a resistive heater so as to promote ion desolvation. The other end of the pipe was connected to a vacuum chamber containing a 6-inch long quadrupole ion guide $q_{\text {ConDuct }}$, operating at 1-2 Torr. Ions focused in the collision quadrupole ion guide were introduced through a skimmer with an orifice diameter of $1 \mathrm{~mm}$. To the left hand side of the T-quadrupole arm, we attached an S-lens type atmosphere-to-vacuum interface $[31,32]$ used in the commercial Velos Orbitrap mass spectrometers (Thermo).

For measurements of the relative ion transmission efficiency of the two ion interfaces attached to the T-quadrupole, we used two pairs of synthetic peptides (AnaSpec, Inc., Fremont, CA, USA) that were either unlabeled or labeled with ${ }^{13} \mathrm{C}$ and ${ }^{15} \mathrm{~N}$ isotopes. One such pair was human angiotensin I peptide. The sequence of the unlabeled peptide (cat. 20627) is DRVYIHPFHL
$\left(\mathrm{MM}_{\mathrm{avg}}=1296.5 \mathrm{u}\right)$, whereas that for the labeled version (cat. $65140)$ is $\operatorname{DRV}\left(5^{13} \mathrm{C}^{15} \mathrm{~N}\right) \mathrm{YI}\left(6^{13} \mathrm{C}{ }^{15} \mathrm{~N}\right)$ HPFHL $\left(\mathrm{MM}_{\mathrm{avg}}=\right.$ $1309.5 \mathrm{u}$ ). The second peptide pair was the $\beta$-amyloid peptide, fragment 1-15 with the sequences for the unlabeled version (cat. 61798) as DAEFRHDSGYEVHHQ $\left(\mathrm{MM}_{\mathrm{avg}}=1826.9 \mathrm{u}\right)$ and for the labeled version (cat. 61798) as $\mathrm{DA}\left(3^{13} \mathrm{C}{ }^{15} \mathrm{~N}\right) \operatorname{EFR}\left(6^{13} \mathrm{C}\right.$ $\left.4^{15} \mathrm{~N}\right) \operatorname{HDSG}\left(2^{13} \mathrm{C}{ }^{15} \mathrm{~N}\right)$ YEVHHQ $\left(\mathrm{MM}_{\mathrm{avg}}=1843.9 \mathrm{u}\right)$, respectively.

\section{Results and Discussion}

\section{Transmission Properties of ESI Currents Through the ConDuct Inlet Electrode}

During our investigations of the possibility of improving ion transmission of metal capillaries by electropolishing their inside 
surfaces - i.e., to increase the Reynold's number at which the flow in the capillary can be maintained laminar - we noticed that some of the capillaries subsequent to electropolishing transmitted slightly larger ion currents from one end relative to the other. We hypothesized that this effect originated from the formation of a tapered bore during the electropolishing process [33], and that more ions were transmitted when they entered from the narrower end of the capillary. To test this hypothesis, we thought to use an electrode with a well-defined tapered channel and found that a microliter pipette tip made from conductive plastic contained a well-defined, slowly diverging conical channel at the entrance of the pipette tip (Figure 1). When we tested the pipette tip as an electrode through which ions entered the vacuum, we observed close to $100 \%$ ion transmission efficiency when the ions entered the tip of the electrode at its narrowest end.

We compared the transmission efficiency of electrosprayed ions through different types of inlet electrodes (Figure 1b-d) while varying the three parameters most commonly optimized in ESI MS experiments, namely, the ESI voltage, the distance between the ESI emitter tip and the inlet to the mass spectrometer, and the diameter of the tip of the ESI emitter. Figure 2a shows the dependence of the total ion current, the transmitted ion current, and the current loss during transmission on the ESI voltage applied to a $15-\mu \mathrm{m}$ nanospray emitter tip. The measurements of the current were performed using the setup shown in Figure 1a, while electrospraying a solution of $60 \% \mathrm{v}$ methanol and $1 \% \mathrm{v}$ acetic acid in water delivered to the tip at a flow rate of $450 \mathrm{~nL} /$ min. Almost $100 \%$ of the ESI ion current was transmitted into the vacuum over the full range of applied voltages, at least when the tip of the nanospray emitter was positioned approximately $1 \mathrm{~mm}$ from the inlet of the ConDuct. Figure $2 b$ illustrates the behavior of the ion currents when the tip was moved away from the inlet (along an axis connecting the tip of the emitter and the inlet), when the ESI voltage was kept constant at $1500 \mathrm{~V}$.

When we used nanospray emitters with smaller tip diameters, we noticed that the efficiency of the transmitted ion current dropped below $100 \%$, decreasing when the ESI voltage became larger than some threshold value. Figure $2 \mathrm{c}$ shows the
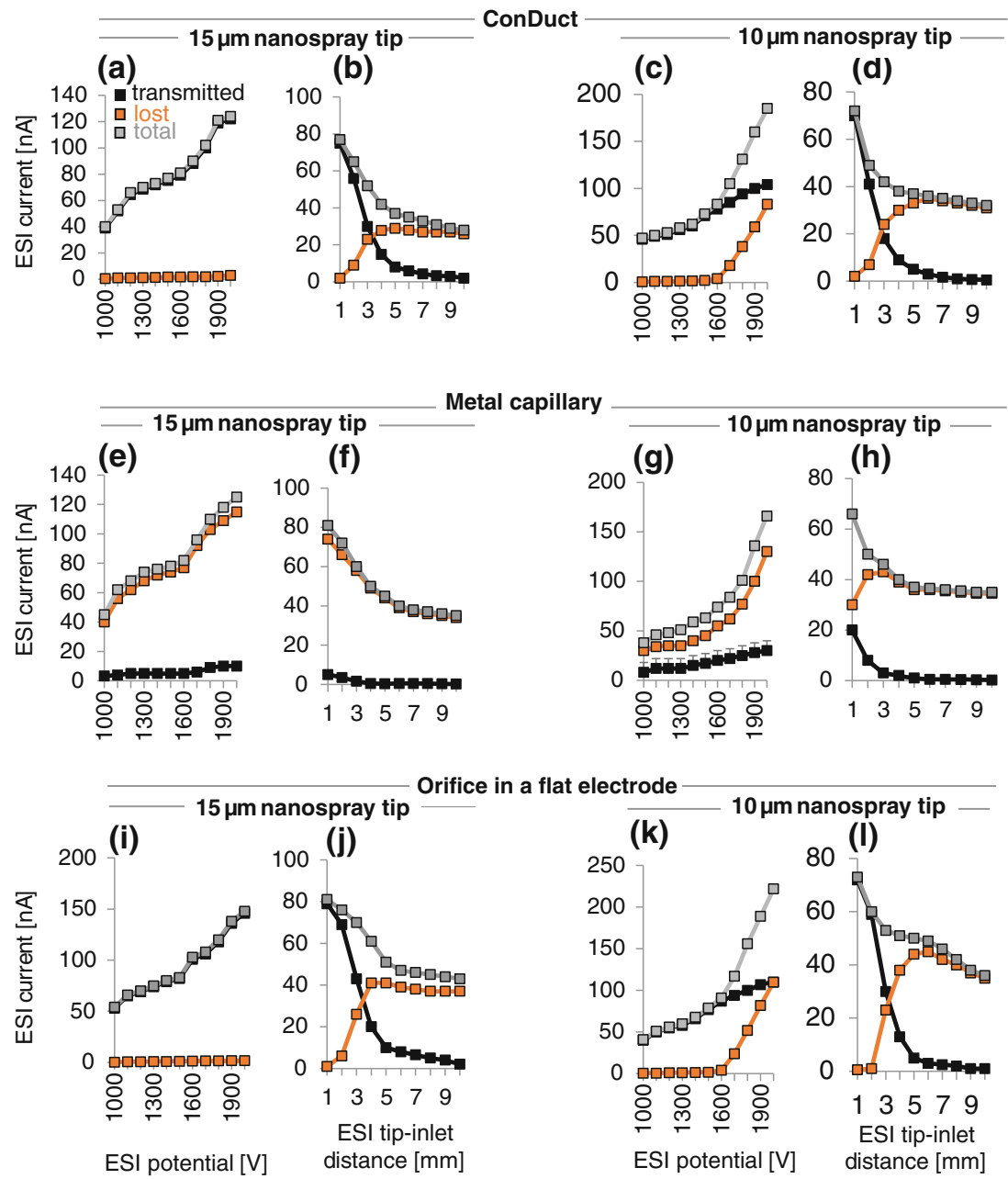

Figure 2. Measurements of the transmitted current (black), total ion current (grey), and current lost (orange) for the ConDuct electrode (a)-(d), metal capillary from a Thermo Velos mass spectrometer (e)-(h), and thin flat electrode (0.06 mm) (i)-(j) with a 0.6$\mathrm{mm}$ orifice, as a function of ESI potential, distance between the electrospray emitter and the inlet, and the diameter of the tip of the nanospray emitter (15- $\mu \mathrm{m}$ nanospray tip and 10- $\mu \mathrm{m}$ nanospray tip) 
transmission through the ConDuct electrode for ions generated by ESI from a $10-\mu \mathrm{m}$ emitter at a flow rate of $150 \mathrm{~nL} / \mathrm{min}$. At voltages greater than $1600 \mathrm{~V}$, the transmitted ion current flattens out, while the total ion current and current losses in the ConDuct continue to grow. One possible explanation for this behavior is a decrease in the efficiency of the flow entrainment capture for the ESI-generated ions species. At these high voltages, the ESI emission jet often transitions into a multi-jet mode, which can generally be avoided by keeping the ESI voltage below $1500 \mathrm{~V}$ for this kind of emitter $[10,12]$. Figure $2 \mathrm{~d}$ illustrates the behavior of the ion current when the $10-\mu \mathrm{m}$ emitter tip was moved away from the inlet while the ESI voltage was kept constant at $1500 \mathrm{~V}$. Figure $2 \mathrm{e}-\mathrm{h}$ show measurements of the total transmitted and ion current losses using a standard 59-mm long metal capillary from a commercial Velos Orbitrap mass spectrometer. The ion current transmitted though this capillary was usually $5 \%$ to $20 \%$ of the total ESI current, with a best value of $28 \%$ observed when the $10-\mu \mathrm{m}$ emitter was positioned $1 \mathrm{~mm}$ from the inlet. These measurements are in agreement with values measured previously by the Smith group [5, 28].

While we observe that the ConDuct electrode is considerably more efficient than the straight metal capillary in transmitting ESI current, a question arises as to what extent the shorter length of the diverging channel $(7 \mathrm{~mm})$ versus the longer metal capillary $(59 \mathrm{~mm})$ contributes to this increase in efficiency. In order to address this question, we cut a $7-\mathrm{mm}$ long piece of $0.5-\mathrm{mm}$ diameter straight metal capillary and compared the ion transmissions with the ConDuct electrode. Indeed, the transmission efficiency of a shorter metal capillary increased to approximately $30 \%$ of the total ESI current despite the very similar gas conductance of these two electrodes, as deduced from the pressure in the vacuum chamber, which was approximately 3.2 Torr for both electrodes. Further shortening of the length of the metal capillary increased the efficiency of transmission, but the progression was very slow (data not shown). Strikingly, however, the ion current transmission through a simple 0.6-mm diameter orifice (Figure 2, panels i-l) was very similar to that through the ConDuct electrode (Figure 2, panels a-d), indicating that approximately $100 \%$ transmission efficiency of the ESI current can be obtained through a simple orifice for a large range of voltages when the tip of the nanospray emitter is $1-2 \mathrm{~mm}$ from the inlet. We next investigated (1) why the ConDuct electrode and the thin flat electrode are both so efficient for transmission of the total ion current, and (2) why we lose such a high fraction of the total ion current to the walls of the metal capillaries.

\section{Suction Properties of the ConDuct Electrode}

The air flow in the vicinity of the inlet of each tested electrode could be different because of corresponding slight differences in gas conductance. The pressure in the first vacuum chamber was approximately 3.2 Torr, 2.8 Torr, and 4.7 Torr, respectively, when using the ConDuct, the metal capillary, and the flat electrode with 0.6 diameter orifice. Although we can estimate the gas velocities at the inlet of each electrode using conservation of mass flow together with pump speed, we decided to perform direct measurements of the velocities of the air flows at the entrance of these three electrodes and observe the influence of the air flow on the shape of the jet emanating from the ESI emitter.

Figure 3 shows the ESI plumes between the nanospray emitter and the entrance orifice for the three types of inlet electrodes. The topmost photographs (Figure 3, panels a, e, and i) were taken with ambient light, and show the position of the tips of the nanospray emitters relative to the inlets. The next row down shows the ESI jets illuminated by green laser light (Figure 3, panels b, f, and j). As a control, we blocked the inlet of each electrode with a piece of thin metal foil while keeping all other parameters in the experiment unchanged (Figure 3, panels $\mathrm{c}, \mathrm{g}$, and $\mathrm{k}$ ). Based on these photographs, we observed that the suction flow was sufficiently strong at the entrance of each electrode to entrain and focus the entire ESI jet into the inlet.

Panels (d), (h), (l) in Figure 3 show photographs of traces of small airborne chalk particles sucked into the ConDuct, the metal capillary, and the orifice in the flat electrode, respectively. To provide the stroboscopic effect, the area in front of each inlet was illuminated by pulses from a light-emitting diode. We measured the length of the most prominent and well-defined traces, which allowed us to determine the velocities of these particles at several distances from the inlet. Then, we divided these velocities by the square of their distances from the inlets and averaged these numbers over several measurements to obtain the average velocity of the particles at a distance of $1 \mathrm{~mm}$ from the inlet. The resulting values are shown in each photograph. The experimentally determined velocities enabled us to calculate the gas conductance of each electrode by estimating how much gas passes through the surface of a 1-mm radius half-sphere around each of the inlets. This also yielded estimates of the maximum velocity of gas at the entrance of each of the inlet electrodes and the corresponding Reynolds number. The data are provided in Table 1.

The estimated velocity of the gas inside the metal capillary was $(71 \pm 18) \mathrm{m} / \mathrm{s}$, resulting in a gas flow with a Reynolds number of $2600 \pm 600$, which is larger than the critical Reynolds number of 2000 for the transition from the laminar to the turbulent regime $[34,35]$. We made another observation that also indicates that the flow in the metal capillary may not be fully laminar. While measuring the transmission efficiency of the ESI current through the capillary, we observed rapid $5-10 \%$ fluctuations in the measured values of both the transmitted and lost ion currents while noticing that their sum (the total ion current) was almost constant. We suggest that this behavior can be explained by the onset of turbulence in the gas flow through the $0.6-\mathrm{mm}$ diameter capillary, which produces fast, correlated fluctuations between the ion current lost on the inner walls of the metal capillary and the transmitted ion current.

Higher Reynolds numbers (4000-7000) characterize the flow of gas through the ConDuct and the orifice in a flat electrode. Although a high Reynolds number may be briefly reached at the "throat" of the orifice, it quickly drops after the inlet electrode [36], with no influence on the ion transmission efficiency. However, the Reynolds number $5400 \pm 1300$ 
(a)

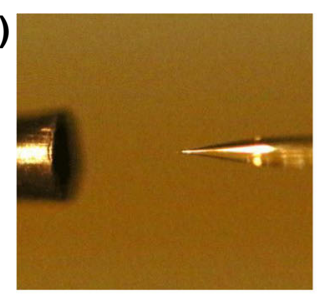

(b)

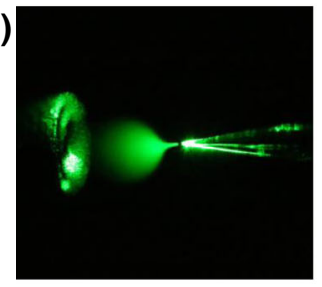

(c)

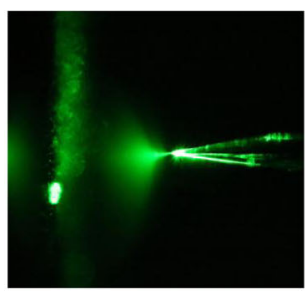

(d)

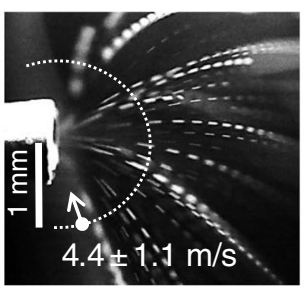

(e)

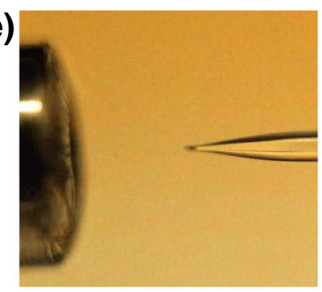

(f)

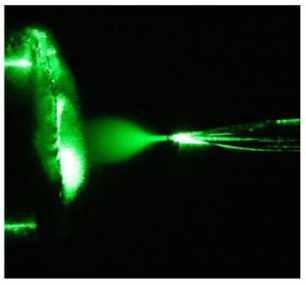

(g)

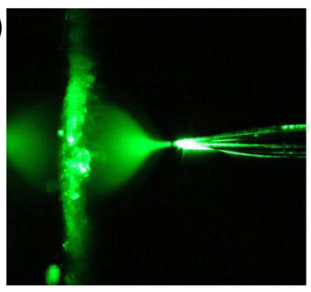

(h)

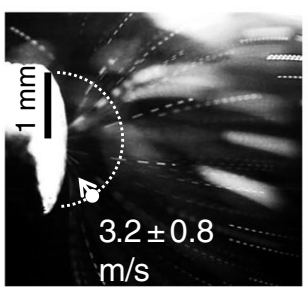

(i)

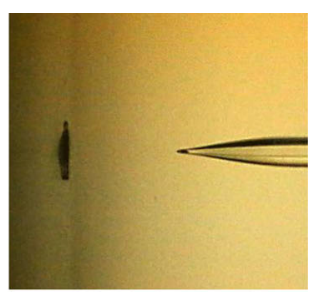

(j)

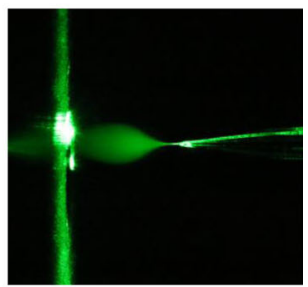

(k)

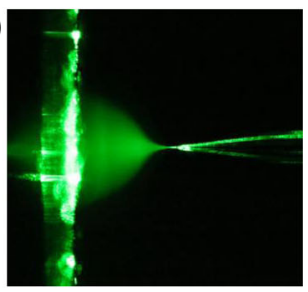

(I)

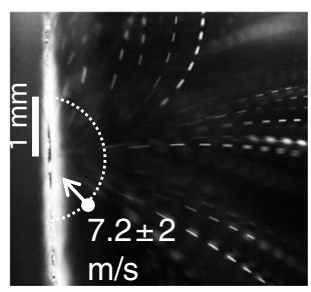

Figure 3. Measurements of the shape of the electrospray jet and the velocity of the suction wind for the ConDuct electrode (a)-(d), a metal capillary (e)-(h) from a Velos Orbitrap mass spectrometer (Thermo), and a 0.6-mm diameter orifice in a thin flat electrode (i)-(l). Panels (a), (e), and (i) show the configurations used when illuminated by ambient light. The distance between the nanospray emitter and the inlet is approximately $1 \mathrm{~mm}$. Panels (b), (f), and (j) show the electrospray jets sucked into the inlets illuminated by green laser light. Panels (c), (g), and (k) show the same electrospray jets but with the inlets blocked by thin metal foils (i.e., with no suction). Panels (d), (h), and (l) show particles of dust being sucked into the inlets. The particles were illuminated by pulses of white LED light with repeating cycles of $50 \mu \mathrm{s}$ on followed by $50 \mu \mathrm{s} \mathrm{off} \mathrm{(10} \mathrm{KHz)} \mathrm{for} \mathrm{(d)} \mathrm{and} \mathrm{(h),} \mathrm{and} 100 \mu \mathrm{s}$ on followed by $100 \mu \mathrm{s} \mathrm{off}(5 \mathrm{KHz})$ for (I). The average measured velocity of the suction wind at a distance of $1 \mathrm{~mm}$ from the inlet is shown in each picture

obtained for a flow of gas in the divergent channel [37-41] of the ConDuct electrode seemingly contradicts our observation of highly focused and directed ion beam from this electrode (see below). One possible explanation comes from the work of
Sparrow et al. [40], who have suggested that even for an initially turbulent regime, the flow of gas in a slow diverging channel may undergo a rapid laminarization by separating from the channel walls. Our next results support this explanation.

Table 1. Characteristics of the Gas Flow Produced by Three Inlet Electrodes

\begin{tabular}{llllll}
\hline & Inlet diameter $[\mathrm{mm}]$ & $\begin{array}{c}\text { Suction velocity }[\mathrm{m} / \mathrm{s}] \\
(1 \mathrm{~mm} \text { from entrance) }\end{array}$ & Conductance $\left[\mathrm{cm}^{3} / \mathrm{s}\right]$ & Velocity at the entrance $[\mathrm{m} / \mathrm{s}]$ & Gas flow Reynolds number R \\
\cline { 2 - 6 } & 0.4 & $4.4 \pm 1.1$ & $28 \pm 7$ & $223 \pm 56$ & $5350 \pm 1340$ \\
ConDuct & 0.6 & $3.2 \pm 0.8$ & $20 \pm 5$ & $71 \pm 18$ & $2560 \pm 640$ \\
Capillary & $7.2 \pm 2.0$ & $47 \pm 17$ & $166 \pm 50$ & $5980 \pm 1500$ \\
Flat electrode & 0.6 & &
\end{tabular}

The Reynolds number for the gas flow through the entrance was calculated according to the equation $R=\frac{d(v) \rho}{\mu}[41]$, where $d$ is the inlet diameter, $v$ is the average velocity at the entrance, $\rho$ is the gas density $\left(1.2 \mathrm{~kg} / \mathrm{m}^{-3}\right)$, and $\mu$ is the kinetic viscosity of the air $\left(2 \times 10^{-5} \mathrm{~kg} /(\mathrm{ms})\right)$. The values are rounded to the nearest 10 . 


\section{Properties of Ion Beams Formed by the ConDuct Electrode}

Figure 4 shows the results of our measurements of the width of the ion beams produced by the different inlet electrodes. To obtain this data, we moved a thin wire probe (Figure 1a) across the ion beam with a spatial resolution of $0.5 \mathrm{~mm}$, and recorded the collected ion current. The ion beam produced by the orifice inlet electrode and the metal capillary were quite divergent, approximately $7^{\circ}$. On the other hand, the ion beam produced by the ConDuct electrode was very narrow, exhibiting an exceptionally low divergence, less than $1^{\circ}$. We also independently determined the beam divergences from these three electrodes by measuring the diameter of the spots left on the flat conductive electrodes that intercepted the ion beam containing molecules of a dye added to the ESI solution [42]. Using this method, we inferred that the ion beam separates from the inner walls of the ConDuct electrode, likely towards the end of the 7-mm conical duct channel (Figure 1b), after which it continues to propagate without significant widening over distances greater than $30 \mathrm{~cm}$, indicating that the flow is highly directed and laminar. We hypothesized that since the beam stays extraordinarily narrow over extended distances, it may be possible to supply desolvation energy to the ions over a long distance using a mixture of heat conduction and radiation. With this hypothesis in mind, we designed our first versions of an atmosphere-tovacuum interface with a ConDuct electrode.

\section{ConDuct Atmosphere-to-Vacuum Ion Interface}

Our first version of the ConDuct-based interface outperformed the original interface from the LCQ DECA mass spectrometer by a factor of more than 400 (Supplementary material). We also wished to investigate how the ConDuct interface performed in comparison with newer, state-of-the-art commercial interfaces. To address this question, we compared the performance of interfaces produced for the Velos Orbitrap and Q Exactive mass spectrometers (Thermo) (henceforth referred to as the S-lens interface $[31,32])$ with a version of the ConDuct interface (Figure 1e).

We began our evaluation of the relative transmission efficiencies of the ConDuct interface versus the commercial S-lens interface using a single ESI source, which we rapidly repositioned between the two entrances. Our initial experiments indicated that both interfaces produced comparable ion fluxes so that it would be desirable to use a more accurate technique for measuring the relative ion transmission efficiency. Thus, we developed a method based on the simultaneous detection of ions produced by two ESI sources, which enter the mass spectrometer through two different interfaces. To distinguish the ion peaks from these two ion sources, we used peptides that were either unlabeled or labeled with heavy isotopes. To further reduce the possibility of systematic errors associated with variations in ion production by the two ESI emitters, we also swapped the positions of the two sources and repeated the measurements.

Figure 5 shows the spectra of the labeled and unlabeled peptides electrosprayed at the inlet of the two interfaces. To

(a) ConDuct

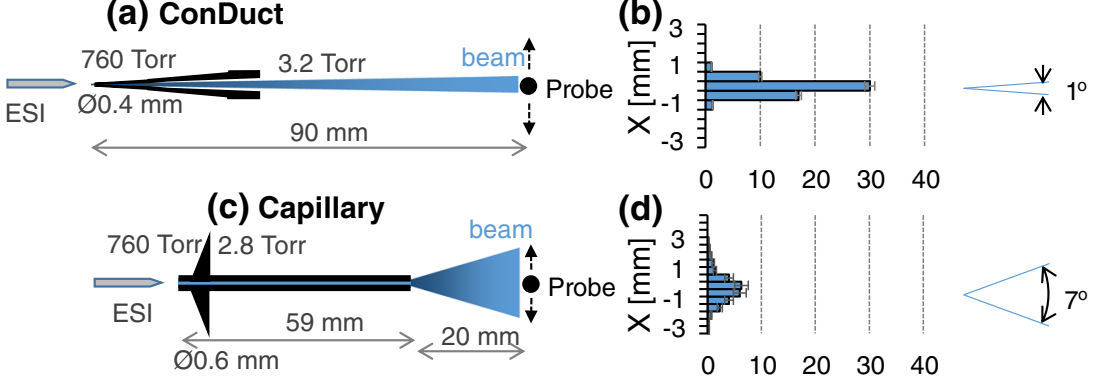

(e) Plate

(f)

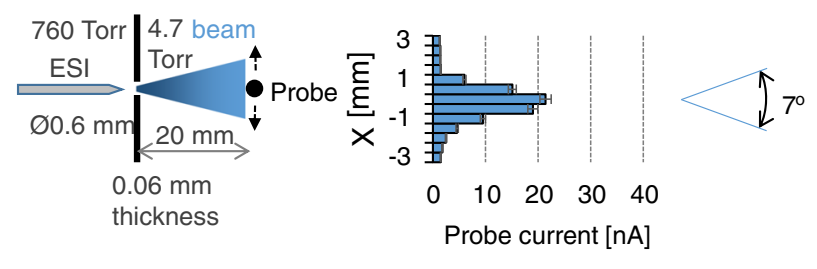

Figure 4. Measurements of the divergence of the ion beams formed by three different inlet electrodes. (a) The ion beam formed by the ConDuct was profiled using a $0.5-\mathrm{mm}$ diameter wire probe positioned $90 \mathrm{~mm}$ from the inlet of the electrode. The most relevant parameters are indicated. (b) Histogram of the current on the probe scanned across the ion beam formed by the ConDuct. (c) Parameters for measurement of the divergence of the beam formed by a metal capillary. (d) Histogram of the current on the probe scanned across the ion beam formed by the capillary. (e) Parameters for measurement of the divergence of the beam formed by the flat electrode with an orifice. (f) Histogram of the current on the probe scanned across the ion beam formed by the orifice in the flat electrode. The average dispersion angle of the ion beam for each case is shown on the right 

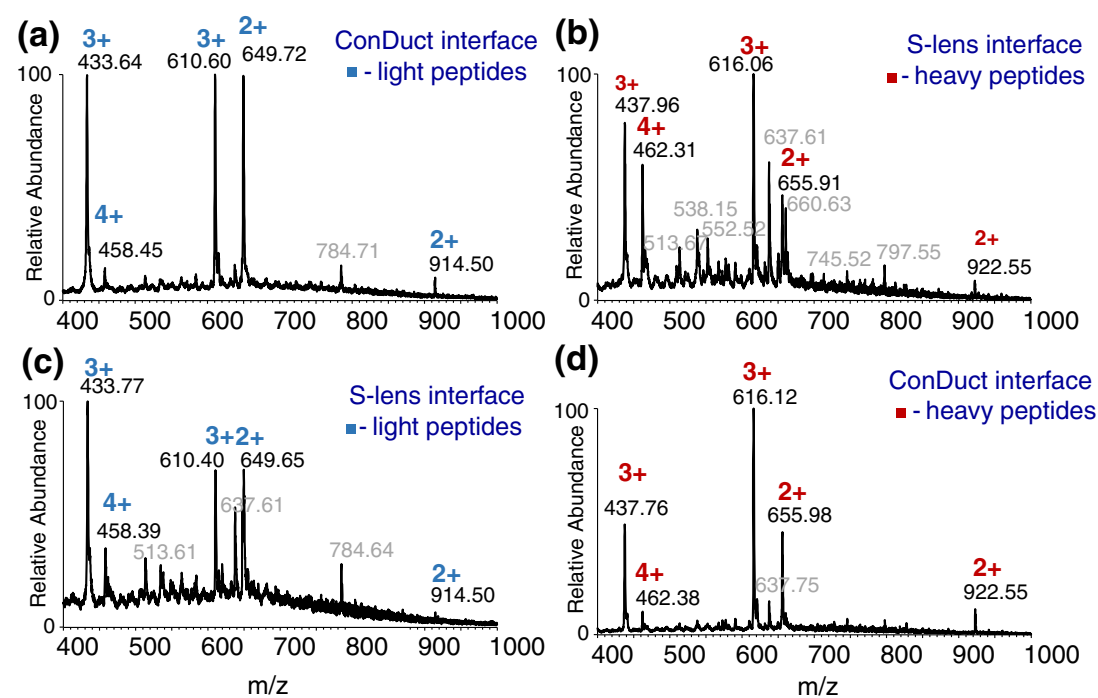

Figure 5. Mass spectra of the unlabeled and heavy-labeled peptides obtained when the nanospray ion sources were positioned in front of either the ConDuct or the S-lens atmosphere-to-vacuum interfaces. (a) Spectra of the two unlabeled peptides angiotensin I $\left(\mathrm{MM}_{\mathrm{avg}}=1296.5 \mathrm{u}\right)$ and $\beta$-amyloid peptide, fragment 1-15 $\left(\mathrm{MM}_{\mathrm{avg}}=1826.9 \mathrm{u}\right)$ obtained by electrospraying an equimolar solution with a concentration of $100 \mathrm{fmol} / \mu \mathrm{l}$ at a flow rate of $150 \mathrm{~nL} / \mathrm{min}$ at the ConDuct interface. (b) Spectra of the same two peptides with some amino acids labeled with heavy isotopes of carbon and nitrogen (see Experimental section). The average MM of the heavylabeled angiotensin was $1309.5 \mathrm{u}$ and the average $\mathrm{MM}$ of the heavy-labeled $\beta$-amyloid peptide was $1843.9 \mathrm{u}$. A $100 \mathrm{fmol} / \mu \mathrm{L}$ solution was electrosprayed at a flow rate of $150 \mathrm{~nL} / \mathrm{min}$ at the S-lens interface. (c) Spectrum of the unlabeled peptides obtained by quickly re-positioning the nanospray ion source in front of the S-lens interface. (d) Spectrum of the heavy-labeled peptides obtained by quickly repositioning the nanospray ion source in front of the ConDuct interface. The charge states of the unlabeled peptides are shown in blue, and labeled peptides are in red. Some prominent impurity peaks are labeled in gray. Note: when the ion trap injection time is very short (a few hundred microseconds), we found that the resulting weak spectra may show different populations of peaks
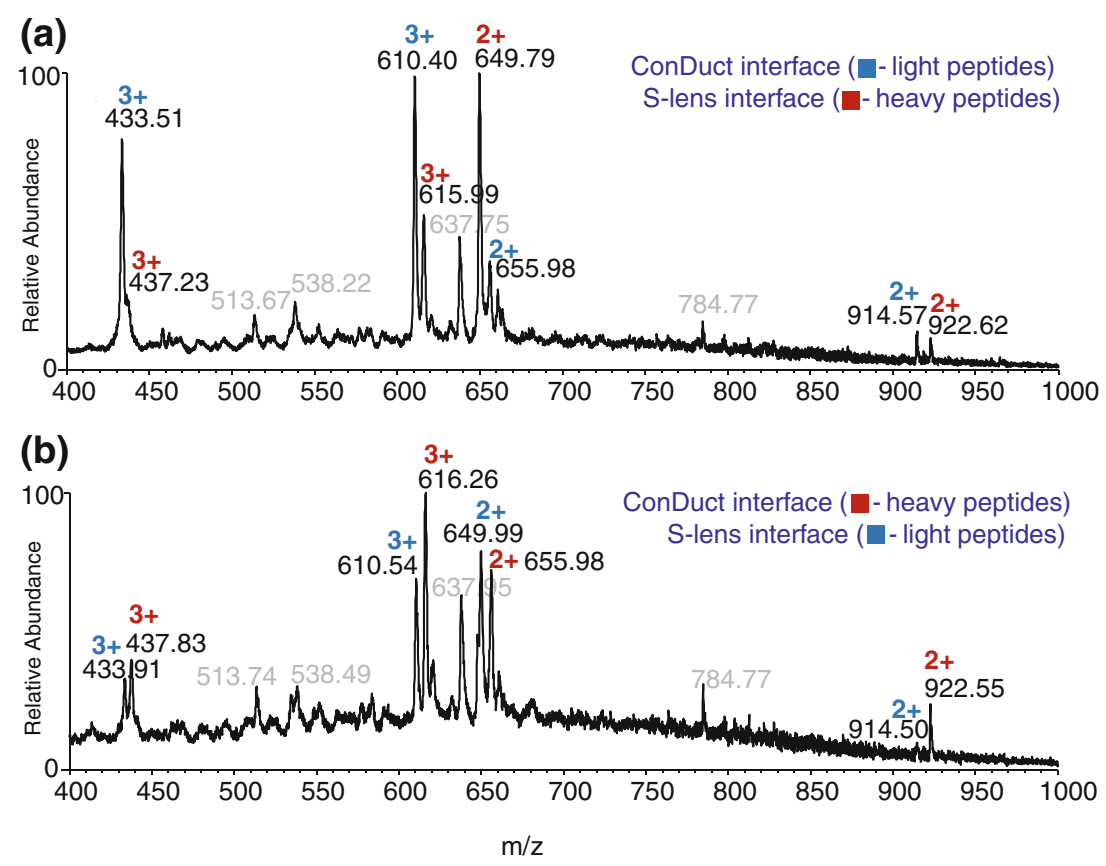

Figure 6. Measurements of the relative transmission efficiencies of the ConDuct and S-lens atmosphere-to-vacuum interfaces. (a) Spectrum of the unlabeled and labeled peptides simultaneously electrosprayed from two identical nanospray ion sources each positioned at one of the two atmosphere-to-vacuum interfaces. Unlabeled peptides were electrosprayed at the ConDuct interface whereas heavy-labeled peptides were electrosprayed at the S-lens interface. (b) The nanospray ion sources were quickly swapped and the measurements repeated. From the ratio of intensities of the charge states pairs, we concluded that the ConDuct interface transmitted 2-3 times more ions than the S-lens interface 
collect the spectra, we first positioned the ion source electrospraying the mixture of the unlabeled peptides at the inlet of the ConDuct interface (Figure 5a). At the same time, the ion source electrospraying the heavy-labeled peptide was positioned far enough from the inlet of the Thermo S-lens interface so that no ions from this source could enter the mass spectrometer. Without interrupting the spray from either ESI emitter, we moved the ion source electrospraying the unlabeled peptides away from the ConDuct interface inlet, positioned the ion source electrospraying the heavy-labeled peptides in front of the S-lens interface inlet, and collected the spectrum shown in Figure $5 \mathrm{~b}$. After that, we swapped positions of the ion sources next to the interfaces and repeated the procedure by engaging only one ion source at a time (Figure 5, panels $\mathrm{c}$ and d).

We note that the exact positioning of the tip of the nanospray emitter relative to the inlet of the ConDuct electrode was not critical for observing a good ion signal as long as the distance between the emitter and the inlet was in the range of 4 to $10 \mathrm{~mm}$. Smaller distances sometimes led to considerable loss of signal, whereas larger distances produced better signal-to-noise ratio in the spectra, but the absolute ion intensity decreased. Positioning of the nanospray emitter near the metal capillary inlet was less critical and the distance was usually set to about $2 \mathrm{~mm}$.

Ions introduced through the ConDuct interface yielded better signal-to-noise ratios than those through the commercial interfaces. Furthermore, the intensity of the peptide ions arriving through the ConDuct interface was higher. Initially, unlabeled peptides were electrosprayed at the ConDuct interface, and heavy-labeled peptides at the S-lens interface (Figure 6a). Then the nanospray ion sources were swapped and the measurements repeated (Figure 6b). From the ratio of the intensities of the pairs of charge states, we found that the ConDuct interface transmitted 2-3 times more ions than the S-lens interface. This result was mirrored by the intensities of pairs of unlabeled and labeled peptide ions in the fragmentation spectra [42].

\section{Conclusions}

The ConDuct inlet electrode exhibits excellent properties as a component of a high-performance atmosphere-to-vacuum ion interface. These properties include high ion transmission efficiency, which under specific conditions can approach $100 \%$. Also, the electrode creates a very narrow, low divergence ion beam that can propagate through long distances into the vacuum without appreciable dispersion. The new atmosphere-tovacuum ion interface that utilizes the ConDuct inlet electrode described here exhibits an improved ion transmission efficiency and signal-to-noise ratio compared with common commercial interfaces based on heated metal capillaries. Importantly, the improvements described in this work were achieved without increasing overall gas conductance through the new interface, which was equipped with the same type of a rotary pump (see Supplementary material) that was originally used to evacuate the commercial LCQ DECA XP interface. Furthermore, our finding that the interface using the ConDuct inlet electrode produces more analytically useful ions than other interfaces even when the tip of the ESI emitter is placed at a distance of 4 $8 \mathrm{~mm}$ from the interface inlet indicates the potential for further improvement of this new interface.

In future work, we will investigate the performance of a series of metal ConDuct electrodes with different angles of channel divergence to maximize further the ion transmission of measurable ions into a mass spectrometer.

\section{Acknowledgments}

This work was supported by the National Institutes of Health (R21 AI096069 and U54 GM103511 to B.T.C.). The authors are grateful to Eloy Wouters and Jean-Jacques Dunyach from Thermo Fisher Scientific for their technical support and helpful discussions.

\section{References}

1. El-Faramawy, A., Siu, K.W.M., Thomson, B.A.: Efficiency of nanoelectrospray ionization. J. Am. Soc. Mass Spectrom. 16(10), 1702-1707 (2005)

2. Schneider, B.B., Javaheri, H., Covey, T.R.: Ion sampling effects under conditions of total solvent consumption. Rapid Commun. Mass Spectrom. 20, 1538-1544 (2006)

3. Geromanos, S., Freckleton, G., Tempst, P.: Tuning of an electrospray ionization source for maximum peptide-ion transmission into a mass spectrometer. Anal. Chem. 72, 777-790 (2000)

4. Cech, N.D., Enke, C.G.: Practical implementations of some recent studies in electrospray ionization. Fundamentals. Mass Spectrom. Rev. 20, 362387 (2001)

5. Page, J.S., Kelly, R.T., Tang, K., Smith, R.D.: Ionization and transmission efficiency in an electrospray ionization-mass spectrometry interface. J. Am. Soc. Mass Spectrom. 18, 1582-1590 (2007)

6. Kim, T., Udseth, H.R., Smith, R.D.: Improved ion transmission from atmospheric pressure to high vacuum using a multi-capillary inlet and electrodynamic ion funnel interface. Anal. Chem. 72, 5014-5019 (2000)

7. Wilm, M.S., Mann, M.: Electrospray and Taylor-cone theory, Dole's beam of macromolecules at last? Int. J. Mass Spectrom. Ion Process 136, 167180 (1994)

8. Wahl, J.H., Goodlett, D.R., Udseth, H.R., Smith, R.D.: Use of smalldiameter capillaries for increasing peptide and protein detection sensitivity in capillary electrophoresis-mass spectrometry. Electrophoresis 14, 448457 (1993)

9. Goodlett, D.R., Wahl, J.H., Udseth, H.R., Smith, R.D.: Reduced elution speed detection for capillary electrophoresis mass-spectrometry. J. Microcolumn 5, 57-62 (1993)

10. Marginean, I., Kelly, R.T., Prior, D.C., LaMarche, B.L., Tang, K., Smith, R.D.: Analytical characterization of the electrospray ion source in the nanoflow regime. Anal. Chem. 80, 6573-6579 (2008)

11. Valaskovic, G.A., McLafferty F.W.: Electrospray ionization source and method of using the same. US Patent 5788166, 4 Aug (1998)

12. Valaskovic, G.A., Murphy III, J.P., Lee, M.S.: Automated orthogonal control system for electrospray ionization. J. Am. Soc. Mass Spectrom. 15, 1201-1215 (2004)

13. Pauly, H.: Atom, molecule, and cluster beams. I. Basic theory, production, and detection of thermal energy beams. Springer series on Atomic, Optical and Plasma Physics. Springer, Berlin, Germany, 1st edition, vol. 28, pp. 344 (2000)

14. Kantrowitz, A., Grey, J.: A high intensity source for the molecular beam. Part I. Theory. Rev. Sci. Instrum. 22, 328 (1951)

15. Yamashita, M., Fenn, J.B.: Electrospray ion source. Another variation on the free-jet theme. J. Phys. Chem. 88(20), 4451-4459 (1984)

16. Thomson. A.B...: Driving high sensitivity in biomolecular MS. Genetic engineering and technology news 32(20), Available at: http:// www.genengnews.com/gen-articles/driving-high-sensitivity-in-biomolecular-ms/4603 (2012). Accessed December 19, 2014 
17. Thomson, A.B...: Declustering and fragmentation of protein ions from an electrospray ion source. J. Am. Soc. Mass Spectrom. 8, 1053-1058 (1997)

18. Fenn, B.J.: Mass spectrometric implications of high-pressure ion sources. Int. J. Mass Spectrom. 200, 459-478 (2000)

19. Cole, R.B. (ed.): Electrospray ionization mass spectrometry: fundamentals, instrumentation, and applications, 1st edition, $600 \mathrm{pp}$. John Wiley and Sons, Inc., New York, USA, 1st edition, pp. 600 (1997)

20. Manisali, I., Chen, D.D.Y., Schneider, B.B.: Electrospray ionization source geometry for mass spectrometry: past, present, and future. Trends Anal. Chem. 25, 243-256 (2006)

21. Chowdhury, S.K., Katta, V., Chait, B.T.: An electrospray-ionization mass spectrometer with new features. Rapid Commun. Mass Spectrom. 4, 81-87 (1990)

22. Lin, B.W., Sunner, J.: Ion transport by viscous gas flow through capillaries. J. Am. Soc. Mass Spectrom. 5, 873-885 (1994)

23. Fenn, J.B., Mann, M., Meng, C.K., Wong, S.F., Whitehouse, G.M.: Electrospray ionization for mass spectrometry of large biomolecules. Science 246, 64-71 (1989)

24. Franzen, J.: Method and device for transport of ions in gas through a capillary. US patent 5736740 A, 7 April 1998

25. Ugarov, M., Perkins, P., Mordehai, A., Barry, B., Li, G., Hansen, S., Stafford, G.: Broad mass range ion transmission and improved peptide detection using QTOF MS equipped with new atmospheric pressure interface. Proceedings of the ASMS Conference, Salt Lake City, Utah, May 23-27 (2010)

26. Shaffer, S.A., Tolmachev, A., Prior, D.C., Anderson, G.A., Udseth, H.R., Smith, R.D.: Characterization of an improved electrodynamic ion funnel interface for electrospray ionization mass spectrometry. Anal. Chem. 15, 2957-2964 (1999)

27. Kelly, R.T., Tolmachev, A.V., Page, J.S., Tang, K., Smith, R.D.: The ion funnel: theory, implementations, and applications. Mass Spectrom. Rev. 29(2), 294-312 (2010)

28. Page, J.S., Marginean, I., Baker, E.S., Kelly, R.T., Tang, K., Smith, R.D.: Biases in ion transmission through an electrospray ionization-mass spectrometry capillary inlet. J. Am. Soc. Mass Spectrom. 20(12), 2265-2272 (2009)

29. Thomson A.B.., Chernushevich I.V., Loboda A.V.: Trapping and processing ions in radio frequency ion guides. In: March R.E., Todd J.F.J. (eds.) Practical Aspects of Trapped Ion Mass Spectrometry. CRC Press, Boca Raton, USA, 1st edition, vol. 10, chap. IV, pp. 525-544 (2010)

30. Chernushevich, I.V.; Loboda, A.V.; Thomson, B.A.; Krutchinsky, A.N.: Mass spectrometer multiple device interface for parallel configuration of multiple devices. US patent 7358488 B2, 15 April 2008
31. Olsen, J.V., Schwartz, J.C., Griep-Raming, J., Nielsen, M.L., Damoc, E., Denisov, E., Lange, O., Remes, P., Taylor, D., Splendore, M., Wouters, E.R., Senko, M., Makarov, A., Mann, M., Horning, S.: A dual pressure linear ion trap Orbitrap instrument with very high sequencing speed. Mol. Cell. Proteom. 8(12), 2759-2769 (2009)

32. Wouters E.R., Splendore, M., Mullen, C., Schwartz J.C., Senko M.W., Dunyach, J-J.: Implementation of a progressively spaced stacked ring ion guide on a linear ion trap mass spectrometer. Thermo Fisher Scientific, San Jose, CA, USA. Available at: http://www.thermoscientific.com/content/ $\mathrm{d} \mathrm{a} \mathrm{m/t} \mathrm{f} \mathrm{s} \mathrm{/} \mathrm{A} \mathrm{T} \mathrm{G} \mathrm{/} \mathrm{C} \mathrm{M} \mathrm{D} \mathrm{/} \mathrm{C} \mathrm{M} \mathrm{D} \mathrm{\%} 20$ D o c u m e n t s / Application $\% 20 \& \% 20$ Technical $\% 20$ Notes/Mass\%20Spectrometry/ $\mathrm{L}$ C \% $20 \mathrm{M} \mathrm{S} / \mathrm{I}$ o n \% $20 \mathrm{Tr}$ a p \% $20 \mathrm{~L} \mathrm{C} \mathrm{\%} 20 \mathrm{M} \mathrm{S} /$ POS_IMSC09_W255_EWouters\%281\%29.pdf. Accessed December 19, 2014

33. Stoffels, J.J., Ells, D.R.: Electropolishing the bore of metal capillary tubes: a technique for adjusting the critical flow. Rev. Sci. Instrum. 50(12), 1574 1578 (1979)

34. Morini, G.L., Lorenzini, M., Salvigni, S., Spiga, M.: Analysis of laminarto-turbulent transition for isothermal gas flows in microchannels. Microfluid Nanofluid 7, 181-190 (2009)

35. Yang, C.-Y., Chen, C.-W., Lin, T.-Y., Kandlikar, S.G.: Heat transfer and friction characteristics of air flow in microtubes. Exp. Thermal Fluid Sci. 37, 12-18 (2012)

36. Bruccoleri, A.R., Leiter, R., Drela, M., Lozano, P.: Experimental effects of nozzle geometry on flow efficiency at low Reynolds numbers. J. Propuls. Power 28(1), 96-105 (2012)

37. Sahu, K.C., Govindarajan, R.: Stability of flow through a slowly diverging pipe. J. Fluid Mech. 531, 325-334 (2005)

38. Eagles, P.M., Weissman, M.A.: On stability of slowly varying flow: the divergent channel. J. Fluid Mech. 69, 241-262 (1975)

39. Dennis, S.C.R., Banks, W.H.H., Drazin, P.G., Zaturska, M.B.: Flow along divergent channel. J. Fluid Mech. 336, 183-202 (1997)

40. Sparrow, E.M., Abraham, J.P., Minkowycz, W.J.: Flow separation in a diverging conical duct: effect of Reynolds number and divergence angle. Int. J. Heat Mass Transf. 52, 3079-3083 (2009)

41. Nakayama, Y. (ed.): Visualized flow: fluid motion in basic and engineering situations revealed by flow visualization. Pergamon Press, Oxford, UK, 1 st edition, vol. 23, pp. 137 (1988)

42. Krutchinsky, A.N., Padovan, J.C., Cohen, H., Chait B.T.: A novel conduct interface for transmitting $\sim 100 \%$ ions from an ESI source into a mass spectrometer. Proceedings of the ASMS Conference, Minneapolis, June 9-13 (2013) 